\title{
DESEMPEÑO FONÉTICO-FONOLÓGICO Y LÉXICO-SEMÁNTICO EN UN GRUPO DE PREESCOLARES DE SECTORES VULNERABLES DE LA PROVINCIA DE CONCEPCIÓN: INCIDENCIA DEL SEXO Y RELACIÓN ENTRE LOS NIVELES LINGÜÍSTICOS
}

\section{PHONETIC-PHONOLOGICAL AND LEXICAL-SEMANTIC PERFORMANCE IN A GROUP OF PRESCHOOLERS FROM VULNERABLE SECTORS OF THE PROVINCE OF CONCEPCIÓN: INCIDENCE OF SEX AND RELATIONSHIP BETWEEN LANGUAGE LEVELS.}

\author{
Nahida Hamdan Rosales \\ Universidad de Concepción \\ Programa de Magíster en Lingüística \\ nahida.hamdan.rosales@gmail.com \\ Jaime Soto-Barba \\ Universidad de Concepción \\ Departamento de Español \\ jaime.soto.barba@udec.cl \\ Katia Sáez-Carrillo \\ Universidad de Concepción \\ Departamento de Estadística \\ ksaez@udec.cl \\ Bernardo Riffo \\ Universidad de Concepción \\ Departamento de Español \\ bernardo@udec.cl
}

\begin{abstract}
Resumen:
El presente trabajo tiene por objetivo conocer el desempeño lingüístico, específicamente fonético-fonológico y léxico-semántico, de un grupo de 30 preescolares que cursan el nivel medio menor en jardines infantiles pertenecientes a una institución educativa de servicio público, y que se ubican en la provincia de Concepción (Chile). En esta investigación, se observó la incidencia del sexo en los resultados obtenidos por los niños y la relación entre el rendimiento presentado por ellos en los dos niveles lingüísticos indicados anteriormente. Con tales propósitos, a los niños se les aplicaron dos instrumentos de evaluación: el TEVI$\mathrm{R}$, con la finalidad de medir el vocabulario pasivo o la comprensión léxica que ellos poseían, y el test TREFF, una prueba a la repetición de palabras, especialmente diseñado para medir el desempeño fonético-fonológico en el habla infantil.

Los resultados de esta investigación indican que un porcentaje importante del grupo de preescolares observados presentó un desempeño lingüístico bajo lo esperado en las dos competencias lingüísticas evaluadas. Por otra parte, los resultados evidenciaron que existen
\end{abstract}


Artículo. Nahida Hamdan Rosales, Jaime Soto-Barba, Katia Sáez-Carrillo, Bernardo Riffo. "Desempeño fonético-fonológico y léxico-semántico en un grupo de preescolares de sectores vulnerables de la provincia de Concepción: incidencia del sexo y relación entre los niveles lingüísticos"

diferencias entre niños y niñas, por cuanto estas últimas manifestaron un mejor rendimiento en los dos niveles lingüísticos considerados en este estudio. Asimismo, los resultados demostraron la existencia de una relación significativa entre el rendimiento de los niños en los dos niveles lingüísticos, es decir, a mayor desempeño fonético-fonológico, mayor desempeño léxico-semántico y, viceversa.

Palabras clave: habla infantil, desempeño fonético-fonológico, desempeño léxicosemántico, TREFF, CLAFF, TEVI-R.

\begin{abstract}
:
The aim of this work is to learn about the linguistic performance, specifically phoneticphonological and lexical-semantic, of a group of 30 pre-school children in the lower middle level in nursery schools that belong to Fundación Integra in the Concepción Province. We observed the incidence of sex in the results obtained by the children and the relationship between their performance in the two linguistic levels mentioned above.

For this purpose, the children were given two evaluation instruments: TEVI-R, with the aim of measuring the passive vocabulary or lexical comprehension that they had, and the TREFF test, a word repetition test, especially designed to measure the phoneticphonological performance in child speech.

The results of this investigation show that a significant percentage of the pre-school group observed showed a linguistic performance below what was expected in the two linguistic competences evaluated. On the other hand, the results showed that there are differences between boys and girls, as the latter showed a better performance in the two linguistic levels considered in this study. Likewise, the results also showed there is a significant relationship between the children's performance in the two linguistic levels, that is, the higher the phonetic-phonological performance, the higher the lexical-semantic performance, and vice versa.
\end{abstract}

Key words: child speech, phonetic-phonological performance, lexical-semantic performance, TREFF, CLAFF, TEVI-R.

Recibido: 23 de septiembre de 2020

Aceptado: 11 de noviembre de 2020 


\section{INTRODUCCIÓN}

El lenguaje constituye una habilidad que se desarrolla en los niños en un tiempo relativamente corto y sin la necesidad de una enseñanza dirigida. Todos los niños en condiciones normales adquieren lenguaje, hecho que demuestra la capacidad innata de la especie humana para adquirir un sistema lingüístico determinado. No obstante, en todos los puntos del desarrollo, los niños difieren en el volumen de su vocabulario, en la complejidad de las estructuras morfosintácticas o fonológicas que producen y en la habilidad global con la cual ellos se comunican (Hoff, 2006). Esta variabilidad manifestada en el proceso de desarrollo del lenguaje muchas veces puede ser el reflejo de diferentes apoyos ambientales.

Tal como lo exponen Rondal y Rodríguez (1985), el valor y la eficacia de la enseñanza implícita que los padres hacen del lenguaje a los niños pueden variar de una pareja de padres a otra y de un medio social a otro, lo que podría constituir uno de los determinantes, al menos parcial, de las diferencias individuales que se observan en la cronología del desarrollo del lenguaje en los niños. En este sentido, numerosas investigaciones han demostrado que una de las variables que se asocia significativamente con el desarrollo del lenguaje en la infancia es el nivel socioeconómico familiar. Al respecto, se ha demostrado que aquellos niños que pertenecen a familias de bajo nivel socioeconómico muestran un desarrollo lingüístico más lento que aquellos niños que pertenecen a familias de un nivel socioeconómico más alto (Hoff, 2003). Consecuentemente, también se ha establecido una relación positiva entre la educación materna y el desarrollo del lenguaje en los niños, es decir, se ha demostrado que los hijos de madres con mayor nivel educacional reportan vocabularios más amplios que los hijos de madres con un nivel educacional menor (Hoff, 2003; Hoff y Tian, 2005).

Algunas investigaciones desarrolladas en el contexto nacional chileno han revelado la misma situación. Por ejemplo, Herrera, Mathiesen y Pandolfi (2000) analizaron la variación en el desarrollo de la comprensión léxica en niños en su etapa preescolar y exploraron algunos factores, ambientales y familiares, que pudiesen encontrarse asociados. Las autoras destacaron la pobreza como un factor relevante, concluyendo que las variables renta per cápita del grupo familiar y el nivel educacional de los padres se asocian fuerte y positivamente con el desarrollo del léxico en los niños, vale decir, mientras más bajo sea el 
Artículo. Nahida Hamdan Rosales, Jaime Soto-Barba, Katia Sáez-Carrillo, Bernardo Riffo. "Desempeño fonético-fonológico y léxico-semántico en un grupo de preescolares de sectores vulnerables de la provincia de Concepción: incidencia del sexo y relación entre los niveles lingüísticos"

nivel socioeconómico, mayor es la probabilidad de que los promedios de desarrollo léxico en los niños sean bajos.

Por otra parte, Pavez, Maggiolo, Peñaloza y Coloma (2009) exploraron el desarrollo fonológico en niños de 3 a 6 años y concluyeron que las variables que incidieron sobre este desarrollo fueron la edad y el nivel socioeconómico. Al analizar únicamente el nivel socioeconómico, los hallazgos de esta investigación indicaron que los niños de nivel medio-bajo presentaron un menor desarrollo fonológico frente a aquellos de niveles medioalto y medio-medio, que no se diferenciaron entre sí. Se corrobora, de esta manera, que la pertenencia a uno de estos niveles socioeconómicos influye de manera clara en el desarrollo del sistema de sonidos lingüísticos, favoreciendo a los niños de nivel alto y sugiriendo que los ambientes más desfavorecidos impactan de modo negativo en el desarrollo fonológico infantil.

Sobre la base de estos hallazgos, esta investigación tiene como primer objetivo determinar cuál es el nivel de desempeño fonético-fonológico y léxico-semántico que presentan los niños que asisten a los jardines infantiles de Fundación Integra, considerando que esta institución chilena ofrece un programa educativo a niños y niñas que tienen entre 3 meses y cuatro años de edad, que viven en situación económica desfavorable y que pertenecen a un sector poblacional de alta vulnerabilidad social (Fundación Integra, 2013).

Por otra parte, el segundo objetivo de esta investigación es observar la incidencia del sexo sobre el desarrollo del lenguaje. Si bien es cierto que existe literatura que sostiene que la aparición y posterior desarrollo del lenguaje suele manifestarse antes en las niñas que en los niños (Garayzábal, 2006; Simonsen, Kristoffersen, Bleses, Wehberg y Jorgensen, 2014; Lange, Euler y Zaretsky, 2016), algunas investigaciones desarrolladas en el contexto nacional sobre el desarrollo léxico y fonológico, específicamente, dan cuenta de resultados variados. Por ejemplo, en el estudio citado en párrafos anteriores sobre la variación en la competencia léxica del preescolar (Herrera, Matiessen y Pandolfi, 2000) los hallazgos indican que los niños presentan promedios mayores que las niñas y que estas diferencias se incrementan y cobran significación al término del año escolar. Por otra parte, en relación con el desarrollo fonológico, las investigaciones indican que no existen diferencias significativas entre niños y niñas, sugiriendo, de esta manera, que el sexo es una variable 
que no afecta el desarrollo fonológico, al menos en el grupo etario considerado en estos estudios (Pavez, Maggiolo, Peñaloza, Coloma, 2009; Coloma, Pavez, Maggiolo, Peñaloza, 2010).

Ahora bien, habitualmente, la tendencia que ha predominado en la investigación sobre la adquisición del lenguaje ha sido la de dividir el estudio de los diferentes componentes del sistema lingüístico, enfocándose en alguno de ellos y otorgando poca atención a la interacción entre ellos. Aunque esta manera de observar los fenómenos lingüísticos ha permitido enriquecer el conocimiento sobre las formas en que los niños adquieren aspectos específicos de su lengua, consecuentemente ha llevado a una falta de atención a las áreas de superposición entre los diferentes componentes que forman el sistema lingüístico (Stoel-Gammon, 2011).

En relación con los niveles lingüísticos de interés del presente estudio, la tendencia observada es la misma. Mientras algunas líneas de investigación se han interesado exclusivamente en cómo se adquieren las palabras, otras se han centrado únicamente en cómo emergen los sonidos del lenguaje, dejando en un segundo plano la interacción entre ambos componentes de la lengua (Storkel y Morrisette, 2002).

No obstante, estudios más recientes han identificado importantes interacciones entre el desarrollo léxico y fonológico temprano, sugiriendo que existe una estrecha relación entre ambos niveles del sistema lingüístico durante el desarrollo del lenguaje en la infancia (Storkel y Morrisette, 2002; Smith, McGregor y Demille, 2006; Stoel-Gamon, 2011; Demuth, 2011; Zamuner, 2011; Kehoe, 2011).

Algunas de las evidencias que dan cuenta de dicha relación fueron indicadas por Hoff y Parra (2011), quienes señalaron que algunos niños producen palabras antes que otros porque son más hábiles en la tarea motora de producción de palabras, con lo cual se demuestra que el orden y el ritmo de adquisición de las palabras se relacionan con la capacidad articulatoria que posee cada niño. Un segundo mecanismo que opera en el niño es el de selección, es decir, resulta más probable que los niños aprendan primero aquellas palabras construidas a partir de sonidos que se encuentran dentro de sus repertorios productivos actuales, frente a aquellas palabras construidas a partir de sonidos que se encuentran fuera de sus repertorios, por lo que el orden de adquisición de las palabras se 
Artículo. Nahida Hamdan Rosales, Jaime Soto-Barba, Katia Sáez-Carrillo, Bernardo Riffo. "Desempeño fonético-fonológico y léxico-semántico en un grupo de preescolares de sectores vulnerables de la provincia de Concepción: incidencia del sexo y relación entre los niveles lingüísticos"

relaciona también con el repertorio fonético que posee el niño en un determinado momento. Por último, algunas palabras son adquiridas por los niños más tarde que otras, porque contienen sonidos que son más difíciles de producir por ellos, o sea, las palabras que son más fáciles, desde el punto de vista fonológico, se adquieren más rápido por los niños y esta capacidad, a su vez, se relaciona estrechamente con la lengua en particular que ellos están adquiriendo. Al respecto, Demuth (2011) comenta que es posible esperar que los niños aprendices de lenguas que poseen estructuras silábicas complejas adquieran los tipos CVC o CCVCC antes que los niños que aprenden una lengua en la cual tales estructuras son menos frecuentes, como sucedió con niños que aprenden inglés como lengua extranjera cuando se les midió su desempeño fonético-fonológico (Fuica y Soto-Barba, 2014).

Hasta ahora se han expuesto algunas de las evidencias que dan cuenta de las influencias fonológicas sobre el desarrollo léxico; sin embargo, también se ha demostrado que el desarrollo léxico impulsa el desarrollo fonológico. En primer lugar, los vocabularios más grandes tienden a hacer uso de un mayor número de sonidos individuales para lo cual se requiere un mayor repertorio articulatorio y, en segundo lugar, en la medida en que los niños aumentan el volumen de sus vocabularios, necesitan de representaciones fonológicas más finas para distinguir y mantener los diferentes elementos léxicos (Hoff y Parra, 2011). Por lo tanto, el desarrollo fonológico se halla favorecido por el incremento léxico que contribuye a una permanente reorganización de la representación fonológica de la palabra en los niños (Shuterland y Guillon, 2005).

Se ha establecido así que la interacción entre el desarrollo léxico y fonológico temprano es cíclica y bidireccional, con una mutua influencia entre ambos componentes del sistema lingüístico. De acuerdo con estas últimas ideas analizadas, esta investigación tiene como tercer objetivo observar la relación existente entre el rendimiento de los niños seleccionados en los dos niveles lingüísticos considerados.

Considerados en su conjunto los antecedentes teóricos y evidencia empírica, los tres objetivos declarados contribuyen a responder las preguntas de investigación que constituyen el problema científico central del presente estudio, a saber, ¿cuál es el grado de desarrollo fonético-fonológico y léxico-semántico que presentan niños en edad pre-escolar 
de sectores vulnerables? ¿incide el sexo en dichos aspectos del desarrollo lingüístico? ¿existe relación entre el desarrollo observado en ambos niveles lingüísticos?

\section{MÉTODOS Y MATERIALES}

2.1. Tipo de estudio: esta investigación correspondió a un abordaje de carácter descriptivo, de corte transversal o sincrónico.

2.2. Selección de los participantes: dentro de la provincia de Concepción (Chile), se seleccionaron 5 jardines infantiles pertenecientes a Fundación Integra. Dado que esta institución plantea públicamente que ofrece un programa educativo a niños entre 3 meses y 4 años de edad, que viven en situación económica desfavorable y de vulnerabilidad social, se asume que en este trabajo la muestra se compone de informantes de nivel socioeconómico bajo. De cada uno de los establecimientos, se seleccionaron 6 niños que cursaban el nivel medio menor, selección que fue llevada cabo por sus educadoras de acuerdo con los siguientes criterios: la edad, procurando que el rango de edad entre ellos se distanciara lo menos posible; la regularidad de asistencia que mantenían; y, por último, que no presentaran trastornos del lenguaje, según los registros con que contaba cada establecimiento. De esta manera, la muestra quedó conformada por un total de 30 niños, en un rango de edad que fue desde los 2 años 7 meses a los 3 años 6 meses.

2.3. Instrumentos: a todos los niños se les aplicaron dos pruebas de lenguaje con la finalidad de conocer su desarrollo lingüístico, tanto a nivel léxico-semántico como fonético-fonológico. Estas pruebas se describen a continuación.

2.3.1. TEVI-R. La medición del nivel léxico-semántico se realizó mediante la aplicación del Test de Vocabulario en Imágenes TEVI-R (Echeverría, Herrera y Segure, 2012), diseñado para evaluar el nivel de comprensión de vocabulario pasivo que posee un sujeto entre 2 y medio y 17 años, hablante nativo del español. Específicamente, esta prueba intenta medir la comprensión que un sujeto tiene de un término que ha sido enunciado 
Artículo. Nahida Hamdan Rosales, Jaime Soto-Barba, Katia Sáez-Carrillo, Bernardo Riffo. "Desempeño fonético-fonológico y léxico-semántico en un grupo de preescolares de sectores vulnerables de la provincia de Concepción: incidencia del sexo y relación entre los niveles lingüísticos"

verbalmente por el examinador; para ello, el sujeto debe seleccionar, de entre cuatro imágenes posibles, aquella que corresponda al término expresado una vez escuchado el estímulo. Este instrumento está conformado por un total de 116 ítemes organizados en orden de dificultad creciente. Por tratarse de un test normado, el TEVI-R permite conocer la posición de un sujeto en relación con sus pares respecto a la comprensión de vocabulario pasivo, estableciendo 5 categorías de desempeño para la interpretación de los resultados que entrega. Estas categorías son: sobresaliente, muy bueno, normal, retraso leve y retraso grave.

2.3.2. TREFF. Los datos para la medición del nivel fonético-fonológico, a su vez, se obtuvieron a través de la aplicación de un test de articulación a la repetición, especialmente diseñado para producir, por lo menos una vez, los fonos o grupos de fonos del repertorio fonético-fonológico del español de Chile, al cual se lo denominó Test de Repetición Fonético-Fonológico (TREFF). De este modo, TREFF, en el caso de las vocales, consideró las 5 vocales en sílaba tónica; todos los diptongos tónicos crecientes y decrecientes; todas las combinaciones vocálicas que generan hiatos en el español. En el caso de las consonantes, exceptuando /r/ que no se manifiesta en inicio de palabra, las 16 consonantes restantes que sí ocurren en este contexto; asimismo, las 17 consonantes en inicio de sílaba en el interior de palabra; las consonantes que se presentan en final de sílaba en interior de palabra, esto es, /p-t-k/ y /b-d - $-\mathrm{g} /, / \mathrm{f} / \mathrm{y} / \mathrm{s} /, / \mathrm{m} / \mathrm{y} / \mathrm{n} /, / 1 / \mathrm{y} / \mathrm{r} /$; las consonantes que se manifiestan en final de palabra, es decir, /d/, / / $/, / \mathrm{x} / / \mathrm{m} /, / \mathrm{n} /, / 1 / \mathrm{y} / \mathrm{r} /$; los grupos consonánticos /pl-pr/, /bl-br/, /tr /, /dr $/, /$ kl-kr/, /gl-gr/, /fl-fr/ en principio de palabra y los mismos grupos en interior de palabra, más $/ \mathrm{t} 1 /$. De esta forma, TREFF quedó constituido por 103 palabras o enunciados que permitieron analizar la producción infantil (en el Anexo 1, se presenta el test TREFF). Es importante indicar que en un trabajo realizado este año el TREFF demostró poseer las propiedades psicométricas de validez y confiabilidad (Vásquez, 2020).

2.4. Procedimientos: ambas pruebas arriba descritas fueron administradas de forma individual, en un lugar apartado de la sala, libre de ruidos molestos y distracciones en 
general. TEVI-R se aplicó siguiendo los procedimientos establecidos en el manual de uso del mismo instrumento, es decir, se le presentaba al participante una lámina con 4 imágenes, al mismo tiempo que el evaluador decía una palabra. El particiante debía indicar a cuál imagen correspondía la palabra, respuesta que era registrada inmediatamente y luego se pasaba a la siguiente lámina. En el caso de TREFF, la aplicación fue grabada en audio con una grabadora digital Tascam, modelo DR-40; con ello, se logró facilitar el posterior análisis de todas las emisiones lingüísticas registradas. Luego, sobre la muestra de habla obtenida, se aplicó la Pauta de Clasificación de Ajustes Fonético Fonológicos o pauta CLAFF (Soto-Barba, León y Torres, 2011) para contabilizar el número de ajustes realizados por cada uno de los niños considerados en el estudio y establecer las principales tendencias a partir de la pronunciación de las clases de sonidos que se produjeron. Una vez aplicada la pauta CLAFF y asignados los puntajes a cada niño, hubo que establecer un procedimiento para interpretar los resultados obtenidos y compararlos de manera similar con los puntajes alcanzados en TEVI-R. Para alcanzar este propósito, se optó por utilizar las mismas 5 categorías de desempeño que utiliza TEVI-R: sobresaliente (entre 0 y 20 ajustes), muy bueno (entre 21 y 40 ajustes), normal (entre 41 y 60 ajustes), retraso leve (entre 61 y 80 ajustes) y retraso grave (81 y más ajustes). Es importante destacar, por último, que en la determinación de la cantidad de ajustes realizados por cada niño y su asociación con un concepto determinado se tuvieron en cuenta los resultados de estudios anteriores realizados con la pauta CLAFF (Fuica y Soto-Barba, 2014; Torres y Soto-Barba, 2016; Torres, León y Figueroa, 2018; León, Soto-Barba y Ñanculeo, 2019; Alarcón, 2019; Lara, Soto-Barba y León), así como también la relación entre puntaje y concepto asociado con el que se interpretan los resultados de la prueba TEPROSIF-R (Pavez, Maggiolo y Coloma, 2009).

\section{RESULTADOS}

Los resultados obtenidos a partir de las pruebas de lenguaje aplicadas a los niños fueron incorporados a una base de datos en planilla Excel, para ser luego analizados con el software estadístico SPSS 23. Se representaron las variables por su promedio y desviación 
Artículo. Nahida Hamdan Rosales, Jaime Soto-Barba, Katia Sáez-Carrillo, Bernardo Riffo. "Desempeño fonético-fonológico y léxico-semántico en un grupo de preescolares de sectores vulnerables de la provincia de Concepción: incidencia del sexo y relación entre los niveles lingüísticos"

estándar. Se utilizó, también, un análisis de varianza (ANOVA) para determinar si existían diferencias significativas entre los resultados que presentaron los niños en relación con la variable sexo. Debido a que la relación fue estadísticamente significativa, se procedió a realizar un test $\mathrm{t}$ de Student para grupos independientes sobre esta misma variable. Por último, para determinar si existía una relación entre el desarrollo fonético-fonológico y léxico-semántico en el grupo de niños seleccionados en esta investigación, se obtuvo el coeficiente de correlación de Pearson. En todos los análisis realizados, se utilizó un nivel de significancia del 0,05 , esto es, cada vez que el valor $\mathrm{p}$ asociado a una prueba fue menor que 0,05 se consideró estadísticamente significativo.

\subsection{Desempeño fonético-fonológico de los niños}

En la tabla de la Figura 1, se puede observar, de manera general, cuál fue la distribución de los 30 niños seleccionados en este estudio en relación con su desempeño fonético-fonológico, una vez aplicada la pauta CLAFF sobre la muestra de habla obtenida a partir de TREFF.

\begin{tabular}{lcc}
\hline INTERPRETACIÓN TREFF & Total & Porcentaje \\
\hline Sobresaliente & 0 & 0 \\
\hline Muy bueno & 4 & 13,33 \\
\hline Normal & 8 & 26,67 \\
\hline Retraso leve & 12 & 40 \\
\hline Retraso grave & 6 & 20 \\
\hline Total & 30 & 100 \\
\hline
\end{tabular}

Figura 1. Tabla con el desempeño fonético-fonológico de los niños.

Tal como se puede apreciar, ningún niño alcanzó un puntaje sobresaliente, aunque este resultado era esperable debido a la corta edad de los niños seleccionados. Un 13,33\% ( 4 casos) obtuvo un rendimiento muy bueno; un 26,67\% ( 8 casos), un rendimiento normal; un 40\% (12 casos) se ubicó en el rango de retraso leve; y un 20\% (6 casos), en el rango de retraso grave. De acuerdo con las categorías establecidas, se observa, entonces, que el 
porcentaje mayor de niños, 60\% (18 casos), se ubicó entre los rangos de retraso leve y retraso grave. Y que el porcentaje restante, 40\% (12 casos), se distribuyó entre las categorías de normal y muy bueno. De esta manera, es posible afirmar que el desarrollo del nivel fonético-fonológico de la mayoría de los párvulos evaluados en este estudio se encuentra descendido en relación con las categorías establecidas.

\subsection{Desempeño léxico-semántico de los niños}

En la tabla de la Figura 2, es posible observar la distribución de los 30 niños evaluados en este estudio en relación con el desempeño que obtuvieron en la prueba de comprensión de vocabulario pasivo, TEVI-R.

\begin{tabular}{lcc}
\hline INTERPRETACIÓN TEVI R & Total & Porcentaje \\
\hline Retraso grave & 6 & 20,0 \\
\hline Retraso leve & 7 & 23,3 \\
\hline Normal & 13 & 43,3 \\
\hline Muy bueno & 3 & 10,0 \\
\hline Sobresaliente & 1 & 3,3 \\
\hline Total & 30 & 100,0
\end{tabular}

Figura 2. Tabla con desempeño léxico-semántico de los niños.

De acuerdo con los resultados, se puede decir que solo un 3,3\% (1 caso) del total de los niños de la muestra obtuvo un desempeño sobresaliente en esta prueba; un 10\% (3 casos) de ellos alcanzó un desempeño muy bueno; un 43,3\% (13 casos) se ubicó dentro del rango de normalidad; un 23,3\% (7 casos), en el rango de retraso leve; y un 20\% (6 casos), en el rango de retraso grave.

Es importante destacar que, en esta prueba, el porcentaje más alto de los niños $43,3 \%$ (13 casos) se ubicó dentro del rango de normalidad en relación con lo esperado para su edad. Este hallazgo indica que a nivel léxico-semántico los niños evidenciaron un mejor desempeño que a nivel fonético-fonológico, donde solo un 26,67\% (8 casos) obtuvo un rendimiento normal. Sin embargo, si se observa al grupo de niños que en esta prueba mostró un desempeño deficiente, es decir, aquellos que se distribuyeron entre los rangos de 
Artículo. Nahida Hamdan Rosales, Jaime Soto-Barba, Katia Sáez-Carrillo, Bernardo Riffo. "Desempeño fonético-fonológico y léxico-semántico en un grupo de preescolares de sectores vulnerables de la provincia de Concepción: incidencia del sexo y relación entre los niveles lingüísticos"

retraso leve y retraso grave, es posible apreciar que la suma de sus porcentajes resulta en el mismo porcentaje de niños distribuidos en el rango normal, o sea, un 43,3\% (13 casos). Esto quiere decir que el número de niños que en esta prueba obtuvo un rendimiento normal es equivalente con el número de niños que se encuentran con un retraso, sea leve o grave, en el desarrollo de la comprensión de vocabulario pasivo. De esta forma, estos resultados nuevamente revelan que una parte importante de los niños evaluados evidencia un desempeño deficiente a nivel léxico-semántico en relación con lo esperado para su edad.

\subsection{Incidencia del sexo}

Con la finalidad de determinar si la variable sexo tenía una incidencia en los resultados obtenidos por los párvulos en las dos pruebas de lenguaje aplicadas, los datos fueron sometidos a análisis mediante la prueba $t$ de Student para grupos independientes. Dicho análisis demostró la existencia de una relación significativa entre el sexo de los informantes y su desempeño lingüístico, tanto en el nivel fonético-fonológico como en el léxico-semántico. La tabla de la Figura 3 muestra cuál fue dicha relación.

\begin{tabular}{|c|c|c|c|c|c|c|c|}
\hline \multirow[b]{2}{*}{ Variable } & \multicolumn{2}{|c|}{ Hombre $(n=11)$} & \multicolumn{2}{|c|}{ Mujer (n=19) } & \multirow[b]{2}{*}{$\mathrm{T}$} & \multirow[b]{2}{*}{$\mathrm{P}$} & \multirow[b]{2}{*}{$\mathrm{h}^{2}$} \\
\hline & Media & D.E. & Media & D.E. & & & \\
\hline TREFF & 77,00 & 27,31 & 59,63 & 19,47 & 2,03 & 0,0520 & 0,1282 \\
\hline TEVI-R & 37,73 & 10,31 & 48,00 & 10,40 & $-2,62$ & 0,0142 & 0,1963 \\
\hline
\end{tabular}

Figura 3. Tabla que nuestra la relación entre sexo de los informantes y desempeño lingüístico.

Como es posible observar, en cuanto a la prueba TREFF, los resultados indican que las niñas presentaron un mejor desempeño que los niños $(59,6(19,5)$ y $77(27,3)$, respectivamente), es decir, realizaron una menor cantidad de ajustes fonético-fonológicos durante la repetición de las 103 palabras evaluadas. Sin embargo, desde el punto de vista estadístico, esta diferencia fue débilmente significativa $(p=0,0520)$. Por otra parte, se observa que en TEVI-R, al igual como ocurrió en TREFF, las niñas mostraron un 
desempeño superior a los niños $(48,0(10,4)$ y 37,7 (10,3), respectivamente), es decir, obtuvieron más aciertos en la selección de los términos que evalúa la prueba y, en este caso, la diferencia resultó estadísticamente significativa $(\mathrm{p}=0,0142)$.

\subsection{Relación entre el desarrollo fonético-fonológico y léxico-semántico en los preescolares}

Para determinar si existía una relación entre el desarrollo fonético-fonológico y léxico-semántico en los niños seleccionados en esta investigación, se procedió a realizar un análisis de correlación entre los resultados obtenidos por ellos en TEVI-R con aquellos que alcanzaron en TREFF. Dicho análisis permitió demostrar la existencia de una correlación negativa entre las dos pruebas de lenguaje aplicadas a los niños $(r=-0,41$ y $p=0,0255)$. Esto quiere decir que ambas pruebas se correlacionan en sentido inverso: a valores altos de una de ellas le corresponden valores bajos de la otra y viceversa. Por lo tanto, mientras mayor fue el puntaje obtenido por un sujeto en TEVI-R, este realizó una menor cantidad de ajustes fonético-fonológicos en la prueba TREFF. Por el contrario, mientras más ajustes realizó un individuo, menos puntaje obtuvo en TEVI-R. Esta situación se explica debido a que ambas pruebas se puntúan de manera inversa, es decir, en TEVI-R, un puntaje alto refleja una mayor comprensión de vocabulario pasivo, puesto que representa la cantidad de aciertos obtenidos por un sujeto en la selección de los términos que evalúa la prueba, mientras que en TREFF, a medida que aumenta el puntaje, más bajo es el rendimiento del participante en este nivel lingüístico, ya que refleja la cantidad de ajustes fonéticofonológicos realizados en la repetición de las palabras evaluadas. 
Artículo. Nahida Hamdan Rosales, Jaime Soto-Barba, Katia Sáez-Carrillo, Bernardo Riffo. "Desempeño fonético-fonológico y léxico-semántico en un grupo de preescolares de sectores vulnerables de la provincia de Concepción: incidencia del sexo y relación entre los niveles lingüísticos"

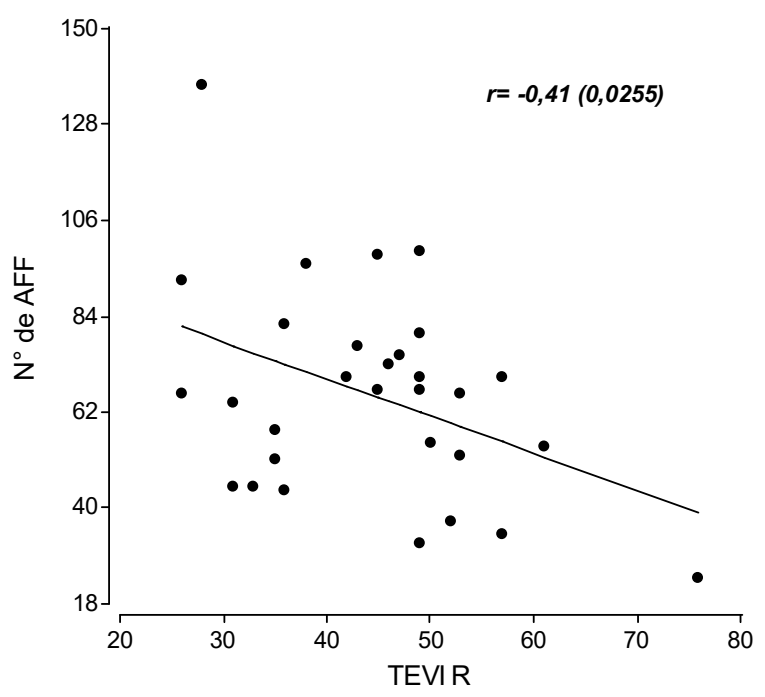

Figura 4. Gráfico que expresa

\section{DISCUSIÓN DE LOS RESULTADOS}

A partir de los resultados obtenidos, es posible afirmar, en primer lugar, que el grupo de preescolares evaluados en este estudio mostró gran variabilidad en relación con su desarrollo lingüístico y que este fenómeno pudo apreciarse tanto a nivel fonéticofonológico como léxico-semántico. No obstante, a pesar de esto último, existe un porcentaje alto del total de niños que mostró un desarrollo lingüístico por debajo de lo esperado. Al comparar el desempeño lingüístico alcanzado por ellos en ambos niveles de análisis, se observan mejores resultados a nivel léxico-semántico.

Por otra parte, los resultados de esta investigación han revelado diferencias entre niños y niñas en cuanto al ritmo de adquisición de las habilidades lingüísticas evaluadas, manifestando las niñas un mejor rendimiento que los niños, tanto a nivel fonéticofonológico como léxico-semántico. Aunque estas diferencias pudieron apreciarse en ambos niveles de análisis, solo fueron significativas a nivel léxico-semántico. Si bien es cierto que otras investigaciones han reportado diferencias a favor de los niños en relación con el desarrollo de la comprensión léxica (Herrera et al., 2000), los resultados recién expuestos son coherentes con buena parte de la literatura que propone que la aparición y posterior desarrollo del lenguaje suele manifestarse antes en las niñas que en los niños (Garayzábal, 
2006; Simonsen et al., 2014; Lange et al., 2016). De esta forma, estos hallazgos complementan la evidencia ya existente en la literatura acerca de la incidencia del sexo sobre la adquisición del lenguaje, sugiriendo que las niñas muestran un desarrollo lingüístico más acelerado, al menos el relacionado con la competencia léxica.

Por último y con respecto al tercer objetivo de esta investigación, los resultados obtenidos permitieron demostrar la existencia de una relación significativa entre el desarrollo fonético-fonológico y léxico-semántico en los preescolares observados: a mayor desempeño fonético-fonológico, mayor desempeño léxico-semántico, y viceversa. Estos resultados son coherentes con la literatura que señala la existencia de una relación estrecha entre ambos niveles lingüísticos durante el desarrollo temprano del lenguaje (Storkel y Morrisette, 2002; Smith, McGregor y Demille, 2006 y Stoel-Gamon, 2011). Sin duda, este es un hallazgo interesante, ya que nos revela información acerca de cómo se comportan ambos niveles lingüísticos durante el periodo de adquisición del lenguaje en la infancia. Parece ser que ambos componentes se van desarrollando de forma paralela y simétrica, es decir, en la medida en que un niño va progresivamente dominando los sonidos de la lengua y sus combinaciones, su comprensión de vocabulario también aumenta, reflejando cierta armonía en el proceso. Por otra parte, debido a que la literatura propone que la interacción entre el desarrollo léxico y fonológico temprano es bidireccional, con una mutua influencia entre ambos componentes del sistema lingüístico (Stoel-Gammon, 2011), resulta lógico esperar que si ambos aspectos del lenguaje se encuentran descendidos, esta retroalimentación no ocurra, o más bien, se produzca en el sentido inverso, enlenteciendo el proceso de adquisición del lenguaje. Lo anterior es especialmente relevante si se considera, nuevamente, que existe un porcentaje importante del grupo de preescolares evaluados en este estudio que mostró bajos resultados en ambos niveles lingüísticos. Pero, además, estos hallazgos pueden considerarse una contribución esperanzadora, en el sentido de que es muy probable que si se refuerza el desarrollo de las habilidades de un nivel lingüístico, se generen avances también en el otro y, viceversa. En síntesis, los resultados recién expuestos, constituyen una nueva evidencia a favor de la hipótesis de una influencia mutua entre el desarrollo fonológico y léxico temprano. La comprobación de esta hipótesis es de especial interés para el área clínica, considerando que puede guiar el diagnóstico y 
Artículo. Nahida Hamdan Rosales, Jaime Soto-Barba, Katia Sáez-Carrillo, Bernardo Riffo. "Desempeño fonético-fonológico y léxico-semántico en un grupo de preescolares de sectores vulnerables de la provincia de Concepción: incidencia del sexo y relación entre los niveles lingüísticos"

tratamiento de los retrasos fonológicos y léxicos del lenguaje (Storkel y Morrisette, 2002). En otro ámbito, teniendo en cuenta el contexto del presente estudio, se cree que estos hallazgos pueden contribuir también al área pedagógica y facilitar herramientas que permitan estimular las habilidades fonológicas y léxicas de los niños en el contexto escolar.

\section{CONCLUSIONES}

Los preescolares observados en este estudio mostraron gran variabilidad en cuanto a sus resultados. De todos modos, existe un porcentaje importante de niños que presentó un rendimiento lingüístico bajo lo esperado en los dos aspectos del lenguaje que fueron evaluados.

Una de las variables que demostró que se asocia con el rendimiento lingüístico de los preescolares evaluados en este estudio fue el sexo. Al respecto, las niñas presentaron un mejor desempeño que los niños en ambos niveles de análisis, siendo esta diferencia más significativa a nivel léxico-semántico.

Finalmente, en el rango de edad de los niños observados, es posible afirmar que existe una relación significativa entre el desempeño obtenido por ellos en los niveles lingüísticos considerados en la investigación, es decir, a mayor rendimiento fonéticofonológico, mayor rendimiento léxico-semántico y viceversa.

\section{FINANCIAMIENTO}

Proyecto VRID Asociativo $\mathrm{N}^{\circ}$ 218.083.036-1.0, financiado por la Universidad de Concepción.

\section{REFERENCIAS}

Alarcón, Enzo. Ajustes fonético-fonológicos en niños de primer año de educación básica provenientes del sistema educativo público y privado de la comuna de San Pedro de la Paz. Incidencia del sexo y el tipo de establecimiento educacional al que asisten. Tesis inédita de Magíster en Lingüística Aplicada. Universidad de Concepción (2019). 
Coloma, Carmen Julia., Pavez, María Mercedes., Maggiolo, Mariangela. y Peñaloza, Christian. "Desarrollo fonológico en niños de 3 y 4 años según la fonología natural: Incidencia de la edad y del género". Signos, 43/72 (2010): 31-48.

Demuth, Katherine. "Interactions between lexical and phonological development: crosslinguistic and contextual considerations". Journal of Child Language, 38 (2011): 69-74.

Echeverría, Max., Herrera, María Olivia. y Segure, Teresa. TEVI-R. Test de vocabulario en imágenes (tercera edición revisada). Concepción, Chile. Editorial Universidad de Concepción (2012).

Fuica, María Angélica. y Soto-Barba, Jaime. "Ajustes fonético-fonológicos en niños(as) de 4 a 5 años que aprenden inglés como segunda lengua”. Literatura y Lingüística, 30 2 (2014): 283-299.

Garayzábal, Elena. "[el pédo de san dóke no tiene daßo]". ¿Rasgos distintivos o dislalia? La Lingüística y la Logopedia: fronteras disciplinarias y disciplinas complementarias. Lingüística Clínica y Logopedia (pp. 21-79). Madrid. Antonio Machado Libros (2006).

Herrera, María Olivia., Mathiesen, María Elena. y Pandolfi, Ana María. "Variación en la competencia léxica del preescolar: Algunos factores asociados". Estudios filológicos, 35 (2000): 61-70.

Hoff, Erika. "The Specificity of Environmental Influence: Socioeconomic Status Affects Early Vocabulary Development Via Maternal Speech". Child Development, 745 (2003): 1368-1378.

Hoff, Erika. "How social contexts support and shape language development". Developmental Review, 26 (2006): 55-88.

Hoff, Erika. y Parra, Marisol. "Mechanisms linking phonological development to lexical development". Journal of Child Language, 38 (2011): 46-50.

Hoff, Erika. y Tian, Chunyan. "Socioeconomic status and cultural influences on language". Journal of Communication Disorders, 38 (2005): 271-278.

Kehoe, Margaret. "Relationships between lexical and phonological development: a look at bilingual children". Journal of Child Language, 38 (2011): 75-81.

Lange, Benjamin, Euler, Harald, \& Zaretsky, Eugen (2016). Sex differences in language competence of 3- to 6-year-old children. Applied Psycholinguistics, 37(6), 14171438. doi:10.1017/S0142716415000624

Lara, Isolina., Soto-Barba, Jaime. y León, Hernán. “Ajustes fonético-fonológicos en niños entre 4 y 5 años de edad de nivel sociocultural bajo". Onomázein (presentado para su evaluación).

León, Hernán., Soto-Barba, Jaime. y Ñanculeo, Marco. “Ajustes fonético-fonológicos en niños de habla típica entre 3 y 4, y entre 4 y 5 años de edad". Onomázein, 43, 2 (2019) 200-222.

Pavez, María Mercedes., Maggiolo, Mariangela. y Coloma, Carmen Julia. Test para evaluar procesos de simplificación fonológica. TEPROSIF-R (tercera edición actualizada). Santiago, Chile. Ediciones Universidad Católica de Chile (2009).

Pavez, María Mercedes., Maggiolo, Mariangela., Peñaloza, Christian. y Coloma, Carmen Julia. "Desarrollo fonológico en niños de 3 a 6 años: incidencia de la edad, el género 
Artículo. Nahida Hamdan Rosales, Jaime Soto-Barba, Katia Sáez-Carrillo, Bernardo Riffo. "Desempeño fonético-fonológico y léxico-semántico en un grupo de preescolares de sectores vulnerables de la provincia de Concepción: incidencia del sexo y relación entre los niveles lingüísticos"

y el nivel socioeconómico”. Revista de Lingüística Teórica y Aplicada, 472 (2009): 89-109.

Reporte. Fundación Integra (2013).

Rondal, Jean. y Rodríguez, Carlos. "Hacia una teoría cognitivo-ambientalista de la adquisición del lenguaje". Revista Mexicana de Análisis de la Conducta, 111 y 2 (1985): 55-68.

Shuterland, Dean. y Guillon, Gail. "Assessment of Phonological Representations in Children with Speech Impairment". Language, Speech and Hearing Services in Schools, 36 (2005): 294-307.

Simonsen, Hanne Gram, Kristoffersen, Kristian E., Bleses. Dorthe, Wehberg, Sonja, Jørgensen, Rune. (2014). The Norwegian Communicative Development Inventories: Reliability, main developmental trends and gender differences. First Language,34 (1): 3-23. doi: $10.1177 / 0142723713510997$

Smith, Bruce., McGregor, Karla. y Demille, Darcie. "Phonological development in lexically precocius 2-year-olds". Applied Psycholinguistics, 27 (2006): 355-375.

Soto-Barba, Jaime., León, Hernán. y Torres, Valeska. "Una propuesta para la clasificación de los ajustes fonético-fonológicos del habla infantil (CLAFF)". Onomázein 231 (2011) 69-79.

Stoel-Gammon, Carol. "Relationships between lexical and phonological development in young children". Journal of Child Language, 38 (2011): 1-34.

Storkel, Holly. y Morrisette, Michele. "The Lexicon and Phonology: Interactions in Language Acquisition”. Language, Speech and Hearing Services in Schools, 33 (2002): 24-37.

Torres, Valeska. y Soto-Barba, Jaime. "Ajustes fonético-fonológicos en niños con trastornos específicos del lenguaje mixto (TEL Mixto)". Onomázein 331 (2016): 69-87.

Torres, Juan Carlos., León, Hernán. y Figueroa, Mauricio. "Desempeño fonéticofonológico en niños con Trastorno del Espectro Autista (TEA) de pre-kínder a tercero básico". Revista Chilena de Fonoaudiología, Vol. 17 (2018): 1-18.

Vásquez, Sandra. Determinación de la validez y fiabilidad del Test de Repetición FonéticoFonológica (TREFF) como instrumento para evaluar el desempeño fonéticofonológico en niños chilenos. Tesis inédita Magíster en Lingüística Aplicada. Universidad de Concepción (2020).

Zamuner, Tania. "Stepping backwards in development: integrating developmental speech perception with lexical and phonological development". Journal of Child Language, 38 (2011): 56-60. 


\section{ANEXOS}

ANEXO 1. TEST DE REPETICIÓN FONÉTICO-FONOLÓGICA (TREFF)

Nombre

Edad

Establecimiento:

Evaluador

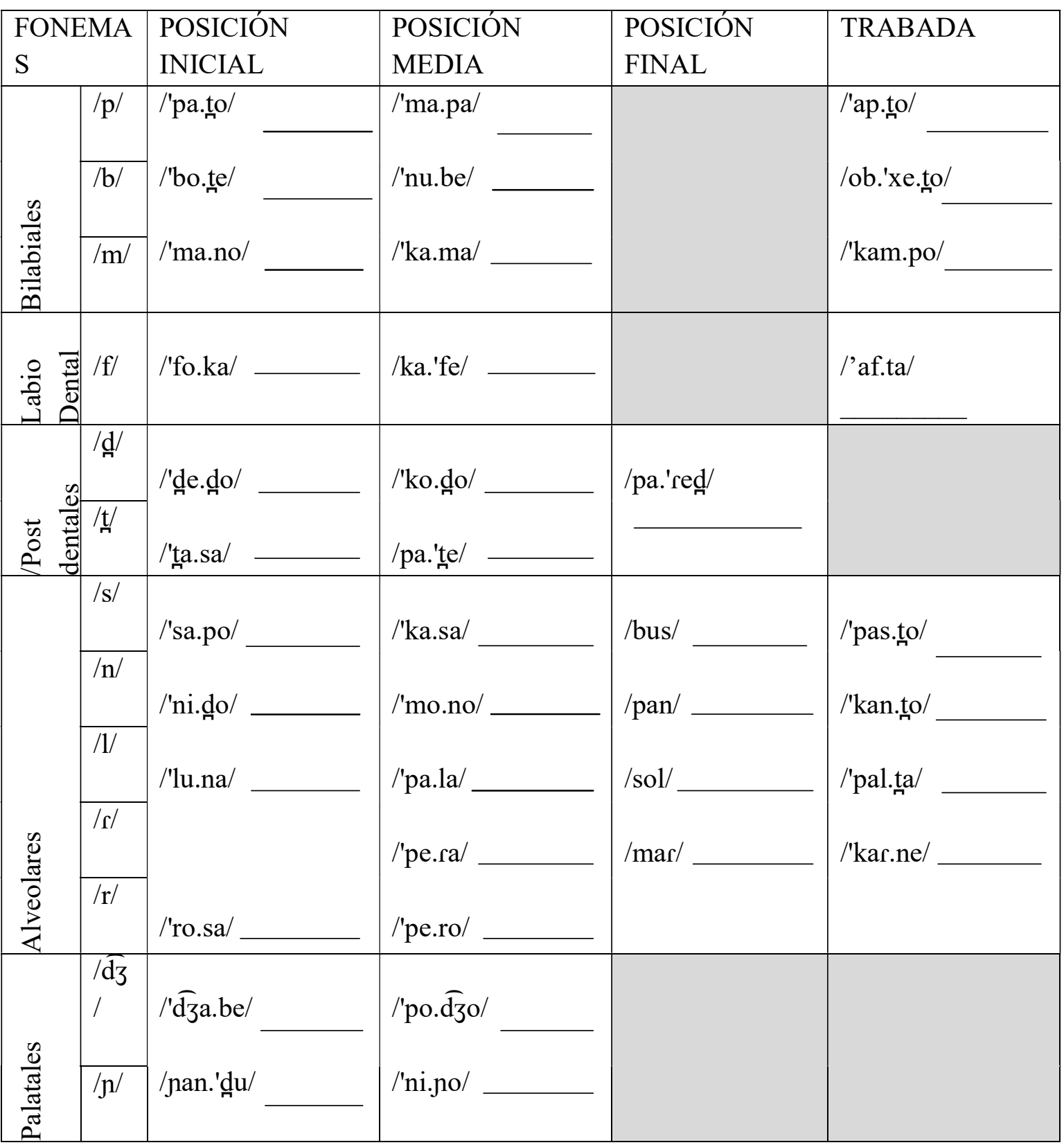

Fecha nacimiento:

Fecha aplicación :
Sexo: 
Artículo. Nahida Hamdan Rosales, Jaime Soto-Barba, Katia Sáez-Carrillo, Bernardo Riffo. "Desempeño fonético-fonológico y léxico-semántico en un grupo de preescolares de sectores vulnerables de la provincia de Concepción: incidencia del sexo y relación entre los niveles lingüísticos"

\begin{tabular}{|c|c|c|c|c|c|}
\hline & $/ \mathrm{t} f /$ & /'t $\int 1 . k o /$ & /'le.tfe/ & & \\
\hline \multirow[b]{3}{*}{$\frac{\infty}{0}$} & $/ \mathrm{k} /$ & \multirow{3}{*}{$\begin{array}{l}\text { /'ka.sa/ } \\
\text { /'ga.to/ }\end{array}$} & \multirow{2}{*}{$\begin{array}{l}\text { /'bo.ka/ } \\
\text { /'xu.go/ }\end{array}$} & & \multirow{3}{*}{$\begin{array}{l}\text { /'kak.tus/ } \\
\text { /'sig.no/ }\end{array}$} \\
\hline & /g/ & & & & \\
\hline & $/ \mathrm{x} /$ & & /'ro.xo/ & /re.'lox/ & \\
\hline
\end{tabular}

\begin{tabular}{|ll|l|}
\hline DIPTONGOS SEMICONSONÁNTICOS & DIPTONGOS SEMIVOCÁLICOS \\
\hline /pie/ & & /rei/ \\
\hline /siu.'dad/ & /'au.to/ \\
\hline /'pio.xo/ & & /'ai.re/ \\
\hline /'ue.bo/ & /'peu.mo/ \\
\hline /'pia.no/ & /'kuo.ta/ \\
\hline /oi/ & & \\
\hline /'kuo.ta & & \\
\hline
\end{tabular}

\begin{tabular}{|l|l|l|l|l|}
\hline \multicolumn{2}{|l|}{ HIATOS } & /ba.'ul/ & /tte.'a.tro/ \\
\hline /'ti.o/ & & & \\
\hline /'pu.a/ & /a.ta.'ud/ & \\
\hline
\end{tabular}




\begin{tabular}{|c|c|c|}
\hline $\begin{array}{l}\text { GRUPOS } \\
\text { CONSONÁNTICOS }\end{array}$ & POSICIÓN INICIAL & POSICIÓN INTERIOR \\
\hline$/ \mathrm{bl} /$ & /'blu.sa/ & /'ka.ble/ \\
\hline$/ \mathrm{pl} /$ & /'plu.ma/ & /a.pla.'nar/ \\
\hline$/ \mathrm{t} \mathrm{l} /$ & & /a.'tle.ta/ \\
\hline$/ \mathrm{kl} /$ & /'kla.bo/ & /'an.kla/ \\
\hline$/ \mathrm{gl} /$ & /'glo.bo/ & /i.'glu/ \\
\hline$/ \mathrm{fl} /$ & /flan/ & \\
\hline$/ \mathrm{br} /$ & /'bra.so/ & /a.'bra.so/ \\
\hline$/ \mathrm{pr} /$ & /'pre.so/ & /'kom.pra/ \\
\hline $\mid \mathrm{tr} /$ & /tren/ & /a.'tras/ \\
\hline$/ \mathrm{d} \mathrm{d} /$ & /dra.'gon/ & /'la.dra/ \\
\hline$/ \mathrm{kr} /$ & /'kre.ma/ & /'mi.kro/ \\
\hline$/ \mathrm{gr}^{\prime} /$ & /'gri.to/ & /'ti.gre/ \\
\hline$/ \mathrm{fr} /$ & /'fru.ta// & /'ko.fre/ \\
\hline
\end{tabular}

\begin{tabular}{|c|c|}
\hline ENUNCIADOS TRISÍLABOS & \\
\hline /to.'ma.te/ & /ko.'ne.xo/ \\
\hline /pa.'le.ta/ _ _ & /xi.'ra.fa/ \\
\hline ENUNCIADOS CUATRÍSILABOS & \\
\hline /ma.ri.'po.sa/ & /tte.'le.fo.no/ \\
\hline /mo.to.' ne.ta/ & /ta.ḑa.' ri.nes/ \\
\hline
\end{tabular}


Artículo. Nahida Hamdan Rosales, Jaime Soto-Barba, Katia Sáez-Carrillo, Bernardo Riffo. "Desempeño fonético-fonológico y léxico-semántico en un grupo de preescolares de sectores vulnerables de la provincia de Concepción: incidencia del sexo y relación entre los niveles lingüísticos"

\begin{tabular}{|l|l|}
\hline ENUNCIADOS PENTASÍLABOS & \\
\hline /ka.ra.bi.'ne.ro/ & /au.to.'ma.tin.ko/ \\
\hline /re.fri.xe.ra.'dor/ & /e.li.'kop.te.ro/ \\
\hline
\end{tabular}

Observaciones:- 